\title{
Mercury in arctic tundra snowpack: temporal and spatial concentration patterns and trace-gas exchanges
}

Yannick Agnan ${ }^{1,2}$, Thomas A. Douglas ${ }^{3}$, Detlev Helmig ${ }^{4}$, Jacques Hueber $^{4}$, Daniel Obrist ${ }^{5,2}$

${ }^{1}$ Milieux Environnementaux, Transferts et Interactions dans les hydrosystèmes et les Sols (METIS), UMR 7619, Sorbonne 5 Universités UPMC-CNRS-EPHE, 4 place Jussieu, F-75252 Paris, France

${ }^{2}$ Division of Atmospheric Sciences, Desert Research Institute, Reno, Nevada 89523, USA

${ }^{3}$ US Army Cold Regions Research and Engineering Laboratory, PO Box 35170, Fort Wainwright, Alaska 99709, USA

${ }^{4}$ Institute of Arctic and Alpine Research, University of Colorado, Boulder, Colorado 80309, USA

${ }^{5}$ Department of Environmental, Earth, and Atmospheric Sciences, University of Massachusetts, Lowell, MA 01854, USA

Correspondence to: Yannick Agnan (yannick.agnan@biogeochimie.fr) and Daniel Obrist (daniel_obrist@uml.edu)

\section{Supplementary material:}

Table S2: Summary total $\left(\mathrm{Hg}_{\text {tot }}\right)$ and dissolved $\mathrm{Hg}\left(\mathrm{Hg}_{\text {diss }}\right)$ concentrations in snowpack and surface snow layers on the tundra and lake at Toolik Field Station.

Figure S1: Snow tower installation over the arctic tundra at Toolik Field Station.

Figure $\mathrm{S} 2: \mathrm{Hg}^{0}$ gas concentrations (3-hours averages) in the atmosphere and snowpack interstitial air (10 and $20 \mathrm{~cm}$ above the ground surface) during a week in spring 2016, including an AMDE, measured at Toolik Field Station. The gray bars indicate nighttime periods. 
Table S1: Geographical coordinates of transect sampling sites.

\begin{tabular}{ccccc}
\hline site & latitude & longitude & elevation (m) & $\begin{array}{c}\text { distance to Dalton } \\
\text { Highway }(\mathbf{m})\end{array}$ \\
\hline transect 1 & $68.7605^{\circ} \mathrm{N}$ & $148.8659^{\circ} \mathrm{W}$ & 501 & 730 \\
transect 2 & $69.0350^{\circ} \mathrm{N}$ & $148.8258^{\circ} \mathrm{W}$ & 400 & 230 \\
transect 3 & $69.4212^{\circ} \mathrm{N}$ & $148.6691^{\circ} \mathrm{W}$ & 333 & 210 \\
transect 4 & $69.5692^{\circ} \mathrm{N}$ & $148.6049^{\circ} \mathrm{W}$ & 145 & 560 \\
transect 5 & $69.6741^{\circ} \mathrm{N}$ & $148.7003^{\circ} \mathrm{W}$ & 118 & 240 \\
transect 6 & $69.8324^{\circ} \mathrm{N}$ & $148.7555^{\circ} \mathrm{W}$ & 84 & 140 \\
transect 7 & $70.0031^{\circ} \mathrm{N}$ & $148.6804^{\circ} \mathrm{W}$ & 46 & 250 \\
transect 8 & $70.1323^{\circ} \mathrm{N}$ & $148.4896^{\circ} \mathrm{W}$ & 21 & 250 \\
\hline
\end{tabular}


Table S2: Summary total $\left(\mathrm{Hg}_{\text {tot }}\right)$ and dissolved $\mathrm{Hg}$ ( $\left.\mathrm{Hg}_{\text {diss }}\right)$ concentrations in snowpack and surface snow layers on the tundra and lake at Toolik Field Station.

\begin{tabular}{|c|c|c|c|c|c|c|}
\hline \multirow{2}{*}{ date } & \multirow{2}{*}{ location } & \multirow{2}{*}{ height $(\mathrm{cm})$} & \multicolumn{2}{|c|}{$\mathrm{Hg}_{\text {tot }}\left(\mathrm{ng} \mathrm{L}^{-1}\right)$} & \multicolumn{2}{|c|}{$\mathrm{Hg}_{\text {diss }}\left(\mathrm{ng} \mathrm{L}^{-1}\right)$} \\
\hline & & & mean & SD $^{\mathbf{a}}$ & mean & $\mathbf{S D}^{\mathbf{a}}$ \\
\hline \multirow{3}{*}{ Oct. $14^{\text {th }} 2014$} & \multirow{3}{*}{ tundra } & 36 & 0.22 & 0.04 & 0.18 & 0.00 \\
\hline & & 25 & 0.24 & 0.04 & 0.14 & 0.08 \\
\hline & & 13 & 0.17 & 0.18 & 0.09 & 0.08 \\
\hline \multirow{3}{*}{ Dec. $7^{\text {th }} 2014$} & \multirow{3}{*}{ tundra } & surface & 0.21 & 0.03 & $<\mathrm{DL}^{\mathrm{b}}$ & - \\
\hline & & 21 & 0.43 & 0.55 & 0.12 & 0.03 \\
\hline & & 11 & 1.06 & 1.30 & 0.16 & 0.06 \\
\hline Dec. $31^{\text {st }} 2014$ & tundra & surface & 0.36 & 0.28 & 0.17 & 0.06 \\
\hline \multirow{6}{*}{ Jan. $26^{\text {th }} 2015$} & \multirow{5}{*}{ tundra } & surface & 0.27 & 0.16 & 0.12 & 0.05 \\
\hline & & 50 & 0.36 & - & 0.26 & - \\
\hline & & 37 & 6.23 & - & $<\mathrm{DL}^{\mathrm{b}}$ & - \\
\hline & & 25 & 0.46 & 0.16 & 0.11 & 0.11 \\
\hline & & 10 & 0.28 & 0.17 & 0.08 & 0.06 \\
\hline & lake & 8 & 0.31 & 0.06 & 0.21 & 0.05 \\
\hline Feb. $20^{\text {th }} 2015$ & tundra & surface & 0.18 & 0.03 & 0.13 & 0.02 \\
\hline \multirow{5}{*}{ Mar. $8^{\text {th }} 2015$} & \multirow{4}{*}{ tundra } & 48 & 0.66 & 0.10 & 0.15 & 0.02 \\
\hline & & 37 & 0.59 & 0.36 & 0.11 & 0.10 \\
\hline & & 25 & 0.19 & 0.03 & 0.08 & 0.05 \\
\hline & & 13 & 0.29 & 0.05 & $<\mathrm{DL}^{\mathrm{b}}$ & - \\
\hline & lake & 11 & 0.74 & 0.02 & $<\mathrm{DL}^{\mathrm{b}}$ & - \\
\hline Apr. $3^{\text {rd }} 2015$ & tundra & surface & - & - & 0.24 & 0.06 \\
\hline \multirow{6}{*}{ Apr. $17^{\text {th }} 2015$} & \multirow{5}{*}{ tundra } & surface & 0.87 & 0.25 & 0.21 & 0.04 \\
\hline & & 35 & 1.03 & 0.45 & 0.28 & 0.01 \\
\hline & & 25 & 0.61 & 0.05 & 0.31 & 0.08 \\
\hline & & 15 & 1.24 & 0.41 & 0.29 & 0.15 \\
\hline & & 5 & 0.91 & 0.62 & 0.33 & 0.22 \\
\hline & lake & 11 & 1.43 & 0.23 & 0.09 & 0.07 \\
\hline
\end{tabular}

a standard deviation

$\mathrm{b}$ below detection limit

“_" no data 
Table S2: Continued.

\begin{tabular}{|c|c|c|c|c|c|c|}
\hline \multirow{2}{*}{ date } & \multirow{2}{*}{ location } & \multirow{2}{*}{ height $(\mathrm{cm})$} & \multicolumn{2}{|c|}{$\mathrm{Hg}_{\text {tot }}\left(\mathrm{ng} \mathrm{L^{-1 }}\right)$} & \multicolumn{2}{|c|}{$\mathrm{Hg}_{\text {diss }}\left(\operatorname{ng} \mathrm{L}^{-1}\right)$} \\
\hline & & & mean & $\mathbf{S D}^{\mathbf{a}}$ & mean & $\mathbf{S D}^{\mathrm{a}}$ \\
\hline Oct. $19^{\text {th }} 2015$ & tundra & surface & 0.32 & 0.03 & 0.12 & 0.07 \\
\hline Nov. $15^{\text {th }} 2015$ & tundra & surface & 0.46 & 0.17 & 0.26 & 0.13 \\
\hline \multirow{5}{*}{ Dec. $5^{\text {th }} 2015$} & \multirow{5}{*}{ tundra } & surface & 0.19 & 0.01 & 0.10 & 0.09 \\
\hline & & 34 & 0.32 & - & 0.12 & - \\
\hline & & 26 & 0.78 & - & 0.23 & - \\
\hline & & 16 & 0.43 & 0.12 & 0.20 & 0.04 \\
\hline & & 8 & 0.37 & 0.08 & 0.27 & 0.13 \\
\hline Jan. $13^{\text {th }} 2016$ & tundra & surface & 0.82 & 0.54 & 0.13 & 0.08 \\
\hline \multirow{6}{*}{ Jan. $29^{\text {th }} 2016$} & \multirow{5}{*}{ tundra } & surface & 0.38 & 0.15 & 0.29 & 0.02 \\
\hline & & 35 & 1.00 & - & 0.18 & - \\
\hline & & 25 & 0.69 & 0.75 & 0.16 & 0.05 \\
\hline & & 15 & 0.56 & 0.18 & 0.19 & 0.05 \\
\hline & & 5 & 0.64 & - & 0.17 & - \\
\hline & lake & 5 & 1.63 & 0.63 & 0.24 & 0.06 \\
\hline Feb. $20^{\text {th }} 2016$ & tundra & surface & 0.60 & 0.15 & 0.24 & 0.08 \\
\hline Mar. $3^{\text {rd }} 2016$ & tundra & surface & 0.41 & 0.04 & 0.33 & 0.05 \\
\hline \multirow{7}{*}{ Mar. $25^{\text {th }} 2016$} & \multirow{6}{*}{ tundra } & surface & 1.01 & 0.07 & 0.49 & 0.03 \\
\hline & & 50 & 0.58 & - & 0.13 & - \\
\hline & & 40 & 0.85 & - & 0.14 & - \\
\hline & & 30 & 0.54 & - & 0.10 & - \\
\hline & & 20 & 0.31 & 0.06 & $<\mathrm{DL}^{\mathrm{b}}$ & - \\
\hline & & 10 & 0.46 & 0.13 & 0.19 & 0.09 \\
\hline & lake & 13 & 0.41 & 0.16 & 0.17 & 0.02 \\
\hline Apr. $2^{\text {nd }} 2016$ & tundra & surface & 1.46 & 0.16 & 1.15 & 0.15 \\
\hline Apr. 13 $3^{\text {th }} 2016$ & tundra & surface & 0.21 & 0.03 & 0.13 & 0.01 \\
\hline \multirow{5}{*}{ May $1^{\text {st }} 2016$} & \multirow{4}{*}{ tundra } & surface & 0.66 & 0.23 & 0.23 & 0.08 \\
\hline & & 33 & 0.21 & 0.12 & 0.12 & 0.11 \\
\hline & & 23 & 1.07 & 1.20 & 0.13 & 0.06 \\
\hline & & 15 & 0.43 & - & 0.13 & - \\
\hline & lake & 13 & 0.24 & 0.07 & 0.12 & 0.11 \\
\hline
\end{tabular}




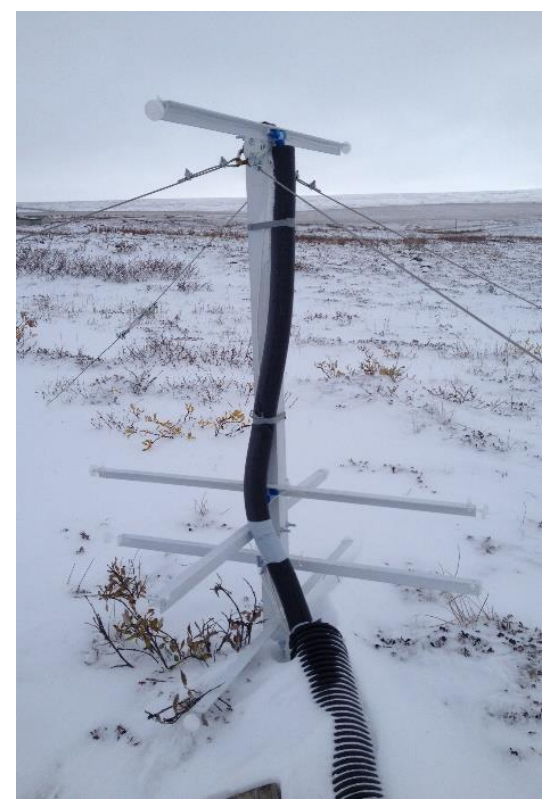

Figure S1: Snow tower installation over the arctic tundra at Toolik Field Station. 


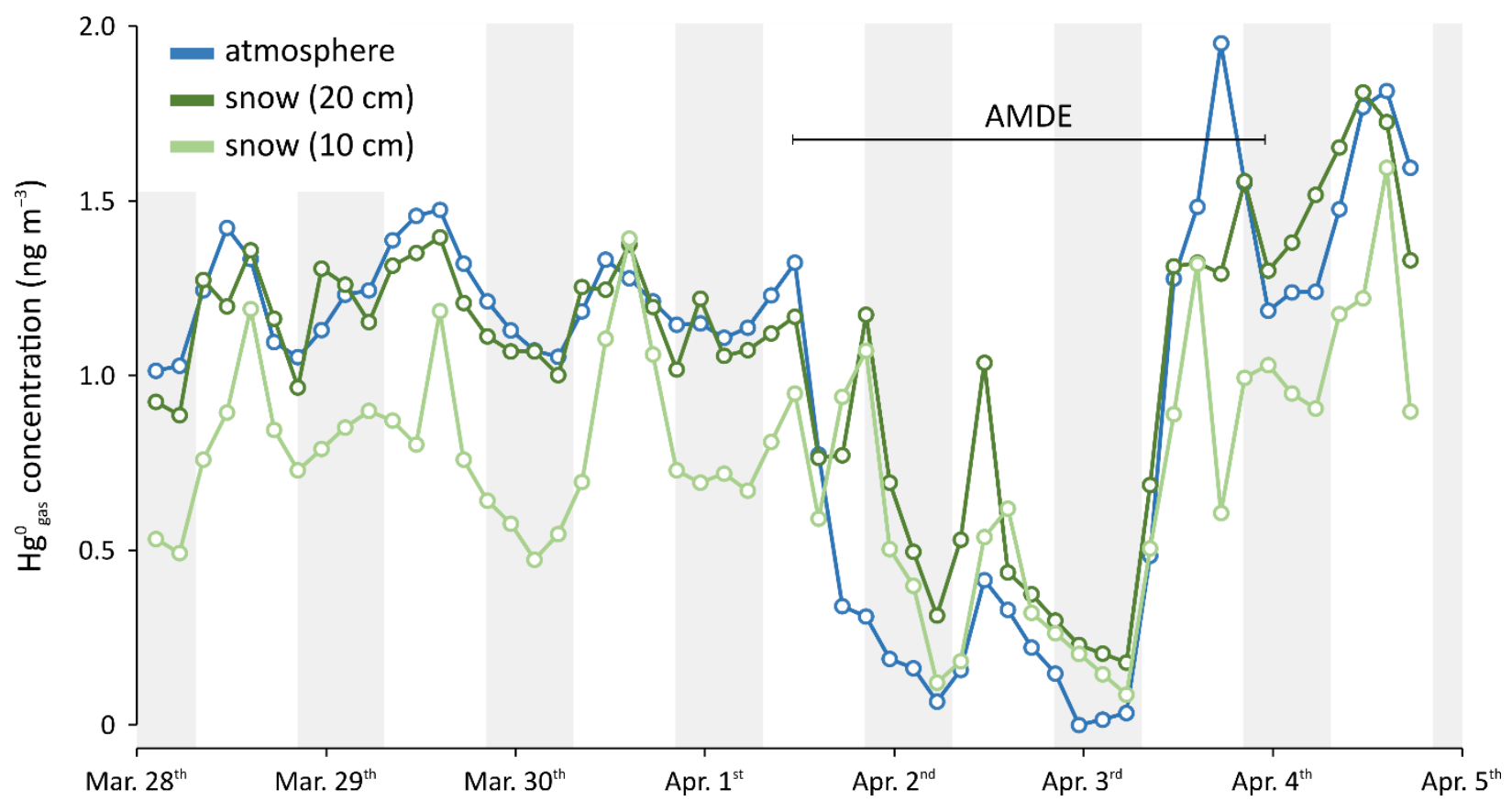

Figure S2: $\mathrm{Hg}^{0}$ gas concentrations (3-hours averages) in the atmosphere and snowpack interstitial air (10 and $20 \mathrm{~cm}$ above the ground surface) during a week in spring 2016, including an AMDE, measured at Toolik Field Station. The gray bars indicate nighttime periods. 